\title{
Two Young Men Who Complained of Voiding Difficulty After Stuffing Odd Materials Into the Urethra to Obtain Sexual Gratification
}

\author{
Sungwoo Hong, Hyung Jee Kim \\ Department of Urology, Dankook University College of Medicine, Cheonan, Korea
}

\begin{abstract}
The self-insertion of foreign bodies into the urethra to obtain sexual gratification is an uncommon paraphilia and may be complicated by their passage into the urethra and subsequent difficulties in recovering the foreign body. There are two key areas of discussion in relation to this issue: (1) the psychological aspects of the action and (2) the possible treatment options. With careful attention to the patient's history, it is usually possible to identify the foreign body and the time since insertion. In most cases, the individual seeks emergency surgical treatment. Radiological evaluation is necessary to determine the exact size, location, and number of objects. Endoscopic retrieval is usually successful, but open surgical removal is required in some cases. We present two cases of self-insertion of foreign bodies into the urethra.
\end{abstract}

Keywords: Foreign bodies; Psychiatric disorders; Urethra

The self-insertion of foreign bodies into the urethra, usually for sexual gratification and autoeroticism, especially during male masturbation, is an unusual but important condition encountered by urologists [1] and has been well documented in the medical literature [2-5]. Common objects used in such cases include fishhooks, metal rods, bones, screws, pellets, electrical cables, and light bulbs $[2,3,6]$. This condition can accidentally occur due to a severe lack of judgment caused by alcohol or drugs, but also arises under normal conditions. Affected patients often present with dysuria, hematuria, urinary retention, and penile pain. In most cases, the inserted foreign bodies cannot be dealt with simply using manual manipulation, but require emergency endoscopic or open surgery. Herein, we present two cases of self-insertion of foreign bodies into the urethra.

\section{CASE REPORTS}

\section{Case 1}

A 36-year-old, married male office worker was admitted to our
Emergency Department (ED) for gross hematuria and severe dysuria caused by the self-insertion of a thick and semirigid electrical cable into his urethral meatus. At first he attempted to remove the cable himself, resulting in further insertion. He first visited a nearby clinic but his urethral pain was not improved and instead deteriorated rapidly following manipulation by the treating clinician. He was still under the influence of alcohol at the time and could not clearly recall what had occurred. On visual inspection, the distal segment of a thick and semirigid electrical cable was protruding through the meatus, and its tip was tied to prevent further advancement into the urinary bladder (Fig. 1A). A plain pelvic radiogram revealed a long radioopaque object, which was twisted multiple times to form a coiled refractory structure in the pelvic cavity (Fig. 1B). We attempted to remove the cable manually, but could not draw it out owing to the severe pain inflicted on the patient. We decided to avoid any further manipulation of the cable owing to the possibility of further urethral injury.

Under general anesthesia, we performed a lower midline in-
Corresponding author: Hyung Jee Kim

Department of Urology, Dankook University College of Medicine,

119 Dandae-ro, Dongnam-gu, Cheonan 330-997, Korea

Tel: +82-41-550-6630 / Fax: +82-41-553-6635 / E-mail: killtumor@naver.com

Submitted: April 24, 2014 / Accepted after revision: May 19, 2014
This is an Open Access article distributed under the terms of the Creative Commons Attribution Non-Commercial License (http://creativecommons.org/licenses/by-nc/3.0/) which permits unrestricted non-commercial use, distribution, and reproduction in any medium, provided the original work is properly cited. 

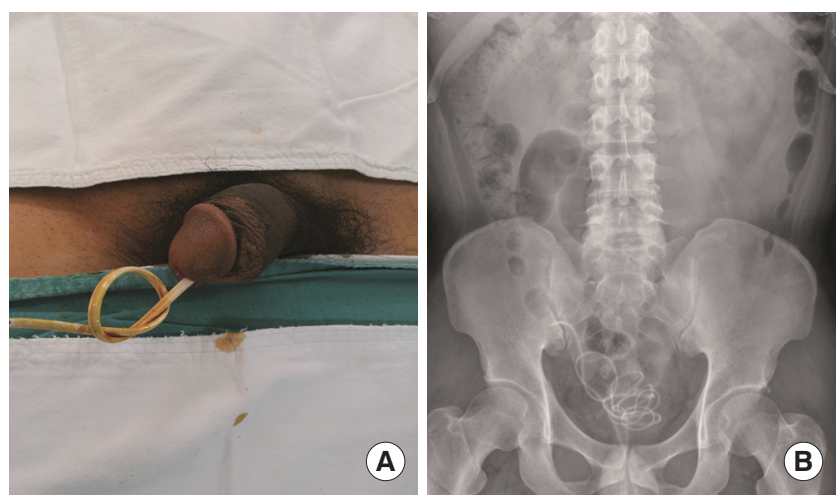

Fig. 1. (A) The distal segment of a thick and semirigid electrical cable protruding through the meatus. Its tip was tied to prevent further advancement into the urinary bladder. (B) A plain pelvic radiograph revealing a long radio-opaque object, which had twisted multiple times to form a coiled refractory structure in the pelvic cavity.

cision and the bladder was opened to a minimum length. The twisted cable was detected in the urinary bladder with no other mucosal injury present, and was gently removed by manipulation towards the head of the patient.

\section{Case 2}

A 22-year-old male presented to our ED complaining of mild lower abdominal pain associated with moderate dysuria. He was an unmarried university student at a Reserve Officers' Training Corps. He was alert and not intoxicated. He clearly explained the circumstances, which led to him inserting bar magnets into his urethra prior to sexual intercourse. He was obsessed with the idea that this would strengthen his penis. However, to eventually retrieve the inserted bar magnet, he inserted another into his urethra. He failed to draw out the two bars with a small circular magnet, and subsequently attempted to remove them using chopsticks. However, this caused the magnets to become more deeply inserted into his urinary bladder.

A plain radiograph of his pelvis revealed what appeared to be two radio-opaque bars and one circular foreign body in his pelvic cavity (Fig. 2A). Under general anesthesia, we examined the region from the urethra to the urinary bladder in the patient using 22-Fr cystoscopy, but failed to retrieve the materials using forceps (Fig. 2B). They were eventually removed endoscopically with grasping forceps.

\section{DISCUSSION}

Most cases of self-inserted foreign bodies are associated with

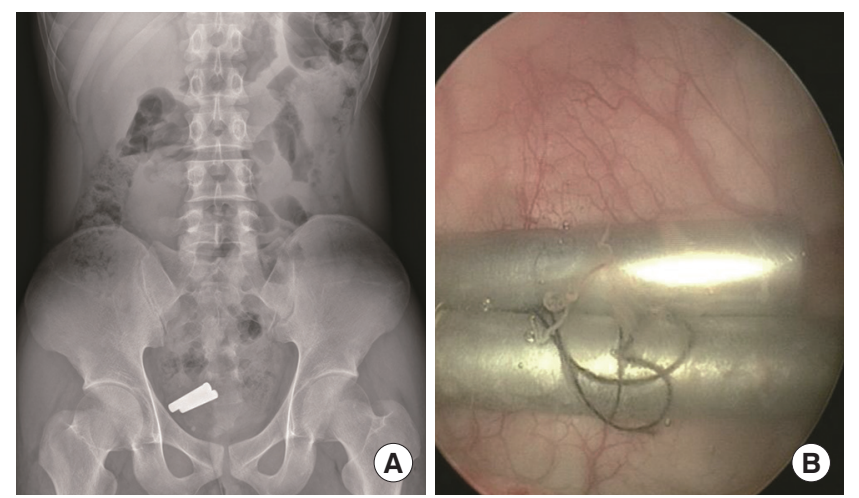

Fig. 2. (A) A plain pelvic radiograph revealing what appeared to be two radio-opaque bars and one circular foreign body in the pelvic cavity of the patient. (B) Two metallic cylinders and one circular magnet stuck together in the urinary bladder are identified using cystoscopy.

autoerotic and sexual gratification, especially during masturbation. Apart from autoerotic stimulation, common motivations for the self-insertion of objects include psychiatric disorders, self-mutilation, intoxication, and curiosity [1-3,5,7-9], or sometimes a combination of these. The Diagnostic and Statistical Manual of Mental Disorders (text revision) defines paraphilia as a mental disorder characterized by recurrent intense sexual urges and sexually arousing fantasies, or behaviors involving inanimate objects [10].

Although much is known about the urologic aspects of genitourinary foreign bodies, the psychiatric profile of an adolescent male who inserts a foreign body into his urethra is currently unavailable. Psychiatric consultations for such self-inserters would enable the development of such a profile and an appropriate management plan [6]. Typical behaviors after insertion often involve shame and embarrassment which often delays the seeking of medical help, sometimes for years [7]. Identification of the true motivation behind these self-insertions is very important because recurrent insertions may occur, or in some cases, there is a nonrecognized mental or organic disease which may even involve forensic implications [3]. For this reason, our two current patients were recommended for psychological counseling before discharge.

The most suitable method for removing a urethral foreign body depends on the size and mobility of the object inserted into the genitourinary tract. When possible, endoscopic and minimally invasive techniques of removal should be used. However, surgical retrieval of a foreign body may be required, particularly when there is a severe associated inflammatory reaction. 
Most foreign bodies in the urethra, especially those below the urogenital diaphragm, can be removed by endoscopy. Foreign bodies in the urethra above the urogenital diaphragm and in the bladder itself can still be removed by endoscopy in many cases, but an open surgical procedure may be required.

In conclusion, self-insertion of foreign bodies into the urethra and/or the urinary bladder is rare. Endoscopic retrieval is usually successful, but a more invasive open surgical procedure is required in some cases. Delayed complications can include urethral stricture disease, and a close follow-up is thus indicated wherever possible. A psychiatric evaluation should also be recommended for all patients.

\section{CONFLICT OF INTEREST}

No potential conflict of interest relevant to this article was reported.

\section{REFERENCES}

1. Stravodimos KG, Koritsiadis G, Koutalellis G. Electrical wire as a foreign body in a male urethra: a case report. J Med Case Rep 2009; $3: 49$.
2. van Ophoven A, deKernion JB. Clinical management of foreign bodies of the genitourinary tract. J Urol 2000;164:274-87.

3. Rahman NU, Elliott SP, McAninch JW. Self-inflicted male urethral foreign body insertion: endoscopic management and complications. BJU Int 2004;94:1051-3.

4. Gonzalgo ML, Chan DY. Endoscopic basket extraction of a urethral foreign body. Urology 2003;62:352.

5. Sukkarieh T, Smaldone M, Shah B. Multiple foreign bodies in the anterior and posterior urethra. Int Braz J Urol 2004;30:219-20.

6. Kenney RD. Adolescent males who insert genitourinary foreign bodies: is psychiatric referral required? Urology 1988;32:127-9.

7. Singh O, Gupta SS. Urethral foreign body causing urethral fistula. Urol J 2012;9:430-2.

8. Nayyar R, Chavda S, Singh P, Gupta NP. Missed diagnosis of anterior urethral valve complicated with a foreign body: a cause for concern. Afr J Paediatr Surg 2011;8:89-91.

9. Trehan RK, Haroon A, Memon S, Turner D. Successful removal of a telephone cable, a foreign body through the urethra into the bladder: a case report. J Med Case Rep 2007;1:153.

10. Woodworth M, Freimuth T, Hutton EL, Carpenter T, Agar AD, Logan M. High-risk sexual offenders: an examination of sexual fantasy, sexual paraphilia, psychopathy, and offence characteristics. Int J Law Psychiatry 2013;36:144-56. 